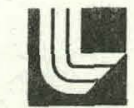 \\ LAWRENCE LIVERMORE LABORATORY \\ University of California/Livermore, California
}

\section{EFFECTS OF SYNCHROTRON RADIATION ON THE COMPRESSION OF A RELATIVISTIC ELECTRON LAYER}

David A. Nowak

January, 1975

NOTICE

This report was prepared as an account of work sponsored by the United States Government. Neither the United States nor the United States Atomic Energy Commission, nor any of their employees, nor any of their contractors, subcontractors, or their employees, makes any warranty, express or implied, or assumes any legal liability or responsibility for the accuracy, completeness or usefulness of any information, apparatus, product or process disclosed, or sepresents that its use would not infringe privately owned rights. 


\section{DISCLAIMER}

This report was prepared as an account of work sponsored by an agency of the United States Government. Neither the United States Government nor any agency Thereof, nor any of their employees, makes any warranty, express or implied, or assumes any legal liability or responsibility for the accuracy, completeness, or usefulness of any information, apparatus, product, or process disclosed, or represents that its use would not infringe privately owned rights. Reference herein to any specific commercial product, process, or service by trade name, trademark, manufacturer, or otherwise does not necessarily constitute or imply its endorsement, recommendation, or favoring by the United States Government or any agency thereof. The views and opinions of authors expressed herein do not necessarily state or reflect those of the United States Government or any agency thereof. 


\section{DISCLAIMER}

Portions of this document may be illegible in electronic image products. Images are produced from the best available original document. 


\title{
EFFECTS OF SYNCHROTRON RADIATION ON THE COMPRESSION OF A RELATIVISTIC ELECTRON LAYER
}

\author{
David A. Nowak \\ University of California, Lawrence Livermore Laboratory \\ Livermore, California 94550 \\ January, 1975
}

\section{INTRODUCT ION}

In this paper we discuss the effect of a changing magnetic field on the equilibrium properties of an infinite electron layer. It is shown that the change in the total energy of the e-layer induced by a changing magnetic field is proportional to the change in the field, if the time scale for the change in the magnetic field is small compared to that for energy loss due to synchrotron radiation. Thus, in principle, it is possible to increase the total energy of an e-layer system by increasing the background magnetic field. The resulting system can be used as an intense source of relativistic electrons of total energy many times the initial e-layer energy. It also provides an intense synchrotron radiation source in the keV range. 1 The change in the field can be induced by compressing a metallic liner enclosing the e-layer or, through the use of a capacitor bank or similar device to increase the background magnetic field. The former has the advantage of confining the magnetic field to the neighborhood of the electron layer.

\section{THEORY}

Here we consider an infinitely long electron layer with thickness small in comparison with the radius, and consisting of $n$ electrons per unit length with electron energy $\mathrm{rmc}^{2}$. Further, we assume the layer 
to be charge neutralized but not current neutralized. Following $G$. Schmidt, ${ }^{2}$ we write the energy of the electrons composing the layer as

$$
r^{2} m^{2} c^{4}=m^{2} c^{4}+p_{z}^{2} c^{2}-\frac{2 q B_{0} P_{\theta} c \gamma^{2}}{(\gamma+v)^{2}}+p_{r}^{2} c^{2}
$$

where $q$ is the charge of the electron $(q=-e), m$ is the mass, $P_{z}$ and $P_{r}$ are the momentum in the axial and radial directions respectively, $P_{\theta}$ is the canonical angular momentum, $B_{0}$ is the confining magnetic field and $\nu$ is equal to $n\left(e^{2} / m c^{2}\right) . P_{z}$ and $P_{\theta}$ are constants of the motion. $P_{r}$ and $P_{z}$ are assumed small in comparison with $\mathrm{rmc}^{2}$. The radius, $R$, of the layer is given by

$$
R=-\frac{(\gamma+v) m c V_{\theta}}{{ }^{q B_{0}}}=\left(\frac{2 P_{\theta} c}{\mathrm{eB}_{0}}\right)^{1 / 2}
$$

where $V_{\theta}$ is the azimuthal velocity, and the field inside the e-layer, $B_{\text {in }}$ is given by

$$
B_{\text {in }}=B_{0} \frac{(\gamma-v)}{\gamma+\nu}
$$

The field reversal parameter $\zeta$ is normally defined as

$$
\zeta=\frac{B_{0}-B_{\text {in }}}{B_{v}}
$$

where $B_{v}$ is the magnetic field present in the absence of the e-layer. In order to relate $B_{v}$ to $B_{0}$ and $B_{i n}$, it is necessary to know the volume of the system. For the purposes here, we will consider the e-layer to be enclosed in a perfectly conducting cylinder of radius $R_{W}$.

The energy per unit length of the electron layer consists of two parts; the kinetic energy per unit length of the layer itself, $E_{L}-n m c^{2}$, and the 
magnetic energy per unit length stored in self-fields $E_{F}$. The total energy per unit length of the system $E_{T}$ can be expressed as

$$
E_{T}=E_{L}+E_{F}+E_{V}
$$

where $E_{V}$ is the energy per unit length of the vacuum magnetic field.

$$
\begin{aligned}
E_{T} & =n \gamma m c^{2}+\left(R_{W}^{2}-R^{2}\right) \frac{B_{0}^{2}}{8}+R^{2} \frac{B_{0}^{2}}{8}\left(\frac{\gamma-\nu}{\gamma+\nu}\right)^{2} \\
& =n \gamma m c^{2}+\frac{R_{W}^{2} B_{0}^{2}}{8}+\frac{R^{2} B_{0}^{2}}{8}\left[\frac{-4 \gamma \nu}{(\gamma+\nu)^{2}}\right]
\end{aligned}
$$

The confining field $B_{0}$ can be calculated by considering the flux contained wi thin the cylindrical system.

$$
R_{W}^{2} B_{v}=\left(R_{W}^{2}-R^{2}\right) B_{0}+R^{2} B_{i n}
$$

Substituting for $R$ and $B_{\text {in }}$ from Eqs. (2) and (3) respectively, we have

$$
B_{0}=\frac{B_{v}}{2}+\frac{B_{v}}{2}\left[1+\frac{m^{2} c^{4}}{e^{2}} \frac{8 v(\gamma+v)\left(\gamma^{2}-1\right)}{\gamma^{2} R_{w}^{2} B_{v}^{2}}\right]^{1 / 2}
$$

If we set $R_{W}^{2}=2 R_{0}^{2}$ where $R_{0}$ is the radius of gyration of an electron of energy $\mathrm{rmc}^{2}$ in a magnetic field $\mathrm{B}_{\mathrm{v}}{ }^{*}$, the total energy of the layer is then given by

$$
\begin{aligned}
E_{L}+E_{F} & =n \gamma m c^{2}-\frac{\gamma v\left(\gamma^{2}-1\right)}{2 \gamma^{2}} \frac{m^{2} c^{4}}{e^{2}} \\
& +\frac{m^{2} c^{4}}{e^{2}}\left(\gamma^{2}-1\right)\left\{\left(\frac{1}{16}\right)\left[1+\left(1+\frac{4 v(\gamma+v)}{\gamma^{2}}\right)^{1 / 2}\right]^{2}-\frac{1}{4}\right\} \\
& =n \gamma m c^{2}\left[1+\frac{v\left(\gamma^{2}-1\right)}{4 \gamma^{3}}\right] \\
& =n r_{0} m c^{2}
\end{aligned}
$$

*This is the special case of constant flux. 
Thus, in principle, it is possible to convert a fraction of the kinetic energy of the layer to magnetic energy.

The energy of the e-layer system can be increased by compressing the system. For a single electron the change in energy is given by

$$
\frac{d\left(\gamma m c^{2}\right)}{d t}=\frac{\gamma m c^{2}}{2 B} \frac{d B}{d t}
$$

This equation is also valid for a current neutralized e-layer in a changing magnetic field. However, in general we have, from differentiating Eq. (1)

$$
\frac{d \gamma m c^{2}}{d t}=\frac{\left(\gamma^{2}-1\right) \gamma(\gamma+v)}{\gamma^{3}+v} \frac{m c^{2}}{2 B_{0}} \frac{d B_{0}}{d t}
$$

The above expression considers only the change in kinetic energy of the electrons due to a change in the confining field. The change in the energy of the electrons plus the energy in the self-fields is physically the important quantity. This can be related to the change in $R_{w}$ by differentiating Eq. (6)

$$
\begin{aligned}
\frac{d E_{L}}{d R_{W}} & =\frac{d E_{T}}{d R_{W}}-\frac{d}{d R_{w}} \frac{R_{w}^{2} B_{v}^{2}}{8} \\
& =\frac{d}{d R_{W}} \frac{m^{2} c^{4}}{e^{2}} \gamma v \frac{\left(\gamma^{2}+1\right)}{2 \gamma^{2}}+2 R_{w} \frac{\left(B_{0}^{2}-B_{v}^{2}\right)}{8} \\
& +\frac{R_{W}^{2}}{8} 2 B_{0} \frac{d B_{0}}{d R_{w}}-2 B_{v} \frac{d B_{v}}{d R_{W}} \\
& =-\frac{B_{0}^{2} R_{W}}{4}-\frac{d}{d R_{w}} \frac{R_{W}^{2} B_{v}^{2}}{8}
\end{aligned}
$$

where $-\frac{B_{0}^{2} R_{W}^{2}}{8} d R_{W}$ is the work done by moving the outer boundary a distance $\mathrm{dR}_{w}$ : The second term on the R.H.S. of Eq. (12) can be evaluated by invoking flux conservation to yield

$$
\frac{d}{d R_{w}} \frac{\left(R_{w}^{2} B_{v}^{2}\right)}{8}=-\frac{R_{w} B_{v}^{2}}{4}
$$


and the change in $E_{L}$ with respect to time is obtained by mutliplying Eq. (13) by $d R_{W} / d t$

$$
\frac{d E_{L}}{d t}=-\left(\frac{B_{0}^{2}-B_{v}^{2}}{4}\right) R_{w} \frac{d R_{W}}{d t}
$$

The above equations are valid for both non-relativistic and relativistic electrons and thus can be used for ion layers as well as electron layers with the appropriate substitutions for $q$ and $\mathrm{m}$. However, if the electron layer is relativistic, it is necessary to consider also the energy loss due to synchrotron radiation. The radiated power, $P$, for a single electron in a magnetic field is given by ${ }^{3}$

$$
P=\frac{-2}{3} \frac{e^{2} c}{a^{2}} \beta^{4} \gamma^{4}=\frac{-2}{3} \frac{e^{4} B^{2}}{m^{2} c^{3}}\left(\gamma^{2}-1\right)
$$

or

$$
\frac{P}{m c^{2}}=-\frac{2}{3} \frac{e^{4} B^{2}}{m^{3} c^{5}}\left(\gamma^{2}-1\right)=-1.937 \times 10^{-9} \mathrm{sec}^{-1} \mathrm{gauss}^{-2} \mathrm{~B}^{2} \gamma^{2}
$$

where $a$ is the local radius of curvature and $B$ is the local magnetic field. It is important to note that the radius, a, which enters into Eq. (15) is not the radius of the layer but the instantaneous radius of curvature, also $\gamma^{2}$ is assumed to be much greater than one. Thus, Eq. (15) must be averaged over the distribution of $\left(1 / a^{2}\right)$. Assuming a linear change in $B$ across the layer, the average power radiated by a single electron is given by (see Appendix B)

$$
\langle P\rangle=-\frac{2}{3} \frac{e^{4} B_{0}^{2}}{m^{2} c^{3}}\left(\gamma^{2}-1\right) \frac{3 \gamma^{2}+\nu^{2}}{3(\gamma+\nu)^{2}}
$$


Thus combining Eqs. (14) and (16) we have

$$
\begin{aligned}
\frac{d E_{L}}{d t}= & \left.\left.\frac{d E_{L}}{d t}\right)_{T}+\frac{d E_{L}}{d t}\right)_{2} \\
= & -\left(B_{0}^{2}-B_{v}^{2}\right) \frac{R_{W}}{4} \frac{d R_{W}}{d t} \\
& -\frac{2}{3} \frac{n e^{4} B_{0}^{2}}{m^{2} c^{3}}\left(\gamma^{2}-1\right) \frac{3 \gamma^{2}+v^{2}}{3(\nu+\gamma)^{2}}
\end{aligned}
$$

The first term on the R.H.S. of Eq. (17) represents the energy change due to compression of the system and is related to the changes in $\gamma, B_{0}$ and R by

$$
\begin{aligned}
\left.\frac{d E_{L}}{d t}\right)_{1} & \left.=\frac{u m^{2} c^{4}}{2 e^{2}}\left(1-\frac{1}{\gamma^{2}}\right) \frac{d \gamma}{d t}\right)_{1}+\frac{R_{W}}{4} B_{0}^{2} \frac{d R_{W}}{d t} \\
& \left.+\frac{R_{W}^{2}}{4} B_{0} \frac{d B_{0}}{d t}\right)_{1}-\frac{B_{v}^{2} R_{W}}{4} \frac{d R_{W}}{d t}
\end{aligned}
$$

while the second term represents the change in energy due to radiation losses and is related to changes in $\gamma$ and $B_{0}$ by

$$
\left.\left.\left.\frac{d E_{L}}{d t}\right)_{2}=\frac{u m^{2} c^{4}}{e^{2}}\left(1-\frac{1}{\gamma^{2}}\right) \frac{d \gamma}{d t}\right)_{2}+\frac{R_{W}^{2}}{4} B_{0} \frac{d B_{0}}{d t}\right)_{2}
$$

The change in $\gamma$ is related to $B_{0}$ by Eq. (11), thus the change in $\gamma$ or $B_{0}$ individually can be found by the appropriate substitution in Eqs. (18) and (19).

If the degradation of the e-layer energy due to radiation losses is neglected, the e-layer behaves like a gas with $\gamma_{G}=3 / 2$ in the limit $\nu \ll \gamma$ and $\gamma_{G}=2$ in the 1 imit $\nu \gg \gamma$, where $\gamma_{G}$ is the ratio of specific heats at constant pressure and constant volume. 
For $d E_{L} / d t$ to be greater than zero, it is necessary, from Eq. (1.7). that

$$
\begin{aligned}
\frac{d R_{w}}{d t} & >\left(\frac{B_{0}^{2}}{B_{0}^{2}-B_{v}^{2}}\right)\left(\frac{8}{3}\right)\left(\frac{3 \gamma^{2}+v^{2}}{3(v+\gamma)^{2}}\right)\left(\gamma^{2}-1\right) v \frac{r_{e}}{R_{w}} c \\
& >\left(\frac{R_{w}^{2}(\gamma+v)^{2}}{R_{w}^{2}(\gamma+v)^{2}-R^{2} v^{2}}\right)\left(\frac{2}{3}\right)\left(\frac{3 \gamma^{2}+v^{2}}{3(\gamma+v)^{2}}\right)(\gamma+v)\left(\gamma^{2}-1\right) \\
& \times\left(\frac{r_{e}}{R_{w}}\right) c\left(\frac{R_{w}}{R}\right)^{2}
\end{aligned}
$$

where $r_{e}$ is the classical electron radius. We can evaluate the above inequality in the limits $\gamma \gg v$ and $\gamma=v$. For $\gamma \gg v$ "weak layer limit" we have

$$
\begin{aligned}
\left.\frac{d R_{W}}{d t}\right)_{\nu=0} & >\frac{2}{3} \gamma\left(\gamma^{2}-1\right)\left(\frac{r_{e}}{R}\right) c\left(\frac{R_{W}}{R}\right) \\
& >5.64 \times 10^{-3}(\mathrm{~cm} / \mathrm{sec}) \frac{\gamma\left(\gamma^{2}-1\right) R_{W}}{R^{2}}
\end{aligned}
$$

This can be related to the critical frequency of the synchrotron radiation spectrum, $W_{c}$, by

$$
\left.\frac{d R_{W}}{d t}\right)_{v=0}>0.95 \times 10^{2}\left(\frac{R_{W}}{R}\right) W_{c}(e V)
$$

If we assume $R_{W}$ to be of the order of $R$ and $d R_{W} / d t$ to be of order $10^{6} \mathrm{~cm} / \mathrm{sec}$, we then find $W_{c}$ to be of order $10^{4} \mathrm{eV}$. In the limit $\gamma=v$ we have

$$
\begin{aligned}
\left.\frac{d R_{w}}{d t}\right)_{\gamma=v} & >\left(\frac{4 R_{W}^{2}}{4 R_{W}^{2}-R^{2}}\right)\left(\frac{4}{9}\right) r\left(\gamma^{2}-1\right) \frac{r_{e}}{R} c \frac{R_{W}}{R} \\
& >\left(\frac{4 R_{W}^{2}}{4 R_{W}^{2}-R^{2}}\right) 0.63 \times 10^{2}\left(\frac{R_{W}}{R}\right) W_{c}(e V)
\end{aligned}
$$


which yields approximately the same upper bound on $W_{c}$ as in the limit $\gamma \gg v$. For the strong layer regime, $\gamma=v$, we have assumed the radius of the electron layer and $\gamma$ to be the same as in the "weak layer limit" $\gamma>\nu$. The effect of the large $v$ is to increase the power emitted per electron in the layer and also to shift the radiation spectrum toward higher frequencies. The radiation spectrum for an electron layer of $\gamma=0$ and $\gamma=\nu$ is plotted in Fig. 1. Also plotted is the black-body spectrum of the same total power as the $\gamma=0$ spectrum and with the peak intensity occurring at the same frequency.

Equation (17) cannot be evaluated without a knowledge of the time dependence of the magnetic field. If we assume the cylindrical wall to be moving inward with a constant velocity, $v$, it is possible to obtain a simple expression for $\gamma$ as a function of time. Defining $T_{0}$ as $T_{0}=r_{0} / v$ where $r_{0}$ is the initial radius of the system boundary, we have

$$
\gamma=\frac{\gamma_{0}^{\lambda}}{\left[1+\alpha\left(\lambda^{4}-1\right)\right]}
$$

where $\alpha=\left(\frac{2}{3}\right) \frac{r_{e}^{2} c}{m c^{2}} \frac{B_{0}^{2} T_{0}^{\gamma_{0}}}{4}$ and $\lambda=\left(1-t / T_{0}\right)^{-1}=r_{0} / r$. In this idealized situation the critical frequency $W_{C}$, in the "weak layer limit" is given by

$$
w_{c}=\frac{w_{c 0} \lambda^{4}}{\left[1+\alpha\left(\lambda^{4}-1\right)\right]^{2}}
$$

In Fig. 2, the critical frequency, $W_{C}$, is plotted as a function of both time, $t / T_{0}$, and the compression, $\lambda$, for various values of $\alpha$. Evaluating the expression for $\alpha$ we find

$$
\alpha=4.85 \times 10^{-10} \mathrm{~B}_{0}^{2} T_{0} \gamma_{0} \sec ^{-1} \text { gauss }^{-2}
$$


Thus it is quite feasible for $\alpha$ to be much less than 1 . If a many-fold increase in the energy of the electron-layer is to be achieved, it is necessary that $\alpha$ be much less than unity.

\section{STABILITY}

The results of the prior section are valid as long as the momentum in the radial direction is small in relation to $\gamma_{\mathrm{mc}}$. The thickness of the layer is related to the maximum radial momentum (see Appendix A) by

$$
\frac{P_{r}^{2}}{2 \gamma m}=1 / 2 \gamma m \omega^{2} d^{2}
$$

where $\omega^{2}=(c / R)^{2}[1+\nu / \gamma(1+R / d)]$, and $2 d$ is the thickness of the layer, $R$ being the radius. Then the restriction on the thickness of the layer becomes

$$
\omega^{2} d^{2}<c^{2}
$$

or for $d \ll R \quad v \geqslant \gamma$ we have

$$
(d / R)(v / \gamma)<1
$$

$\omega$ in Eq. (28) is the frequency of radial oscillations about the mean radius $R$, which is induced by the self-fields of the layer. When the quantity $(d / R)(v / \gamma)$ exceeds one, the electrons can no longer propagate in the azimuthal direction.

Thus there is no upper 1 imit on the current of an infinitely long e-layer, as long as the ratio of $c / R$ is less than $\gamma / v$. However, when the end effects are taken into account, there exists an upper limit to the current per unit length that can be carried by electron layer of finite 
length. (See Appendix A.) This limit can be expressed as

$$
v / \gamma<2
$$

If we allow the plasma to be partially current neutralized, Eqs. $((1)-(4))$ still hold if we replace $v$ by $v_{\text {net }}=v(1-f)$ where $f$ is the fraction of current neutralization. The use of current neutralization allows for e-layer configurations without the presence of large selffields. However, the plasma supporting the return current necessary for current neutralization will drift outward due to the $\underline{J}_{p} \times \underline{B}$ force acting on the plasma. For an equilibrium configuration it is necessary to counteract this force by inducing a pressure gradient in the background plasma across the e-layer. For a fully current neutralized relativistic e-layer, the change in pressure across the e-layer, $\Delta P$, is given by

$$
\Delta P=J_{p} B v 2 d=\frac{\operatorname{necB}_{v}}{2 \pi R}=\frac{B_{v}^{2}}{2 v} \frac{v}{\gamma}
$$

where $2 d$ is the thickness of the e-layer, and $\frac{n e c B v}{2 \pi R}$ is the current per untt length in the e-layer. Thus for $v \geqslant \gamma$ the change in plasma pressure across the e-layer is of the order of the pressure of the vacuum magnetic field. This situation raises serious questions of the stability of such a current neutralized e-layer. One can compare this with the magnetic pressure gradient across a non-neutralized e-layer of the same radius and $\gamma$ as the fully current neutralized e-layer. The change in pressure $\Delta P$ given by

$$
\Delta P=\frac{B_{0}^{2}}{8 \pi}-\frac{B_{i n}^{2}}{8 \pi}
$$

using Eq. (3) we have 


$$
\Delta P=\frac{B^{2}}{2 \pi} \frac{v}{\gamma}
$$

where $B$ is the field that exists in the fully current neutralized e-layer.

\section{DISCUSSION}

In the previous sections we have discussed the compression of a relativistic electron layer and the effect of the synchrotron radiation on the change of energy of the layer induced by the changing magnetic field. In theory, it is possible to increase the total energy many-fold. The limit on the energy increase was shown to be a function of the time for the compression and the rate of the synchrotron radiation losses.

The resulting system can be considered as an intense source of relativistic electrons of much higher energy than presently available from pulsed relativistic electron beam machines. Alternatively, the compressed system would also be an intense source of synchrotron radiation, capable of converting large fractions of the energy of the electron layer into keV photons. It should be noted that the radiation emitted in such a system would be in the plane perpendicular to the axis of the electron layer and thus some care in system design would be required to extract the radiation from the system.

The total energy density capable of being stored in such a system was found to be 1 imited by the effect of the self-fields on the propagation of the current in the azimuthal direction. This is the same phenomenon as the Lawson limit for the maximum current capable of being propagated in a relativistic electron beam machine. ${ }^{4}$ 


\section{APPENDIX A}

Following G. Schmidt, ${ }^{2}$ we write the Hamiltonian as

$$
H=\left[m^{2} c^{4}+P_{z}^{2} c^{2}+\left(\frac{P_{\theta}}{r}-\frac{q}{c} A\right)^{2} c^{2}+P_{r}^{2} c^{2}\right]^{1 / 2}
$$

for $P_{r}^{2} c^{2} \ll \gamma^{2} m^{2} c^{4}$ we have

$$
H \approx \psi+\frac{P_{r}^{2}}{2 \gamma m}=\gamma \mathrm{mc}^{2}
$$

where $\psi$ is given by

$$
\psi=\left[m^{2} c^{4}+P_{z}^{2} c^{2}+\left(P_{\theta} / r-\frac{q}{c} A\right)^{2} c^{2}\right]^{1 / 2} .
$$

The problem can thus be treated as a simple harmonic oscillator in a potential well $\psi$. Assuming a uniform current distribution across an e-layer with inner radius $a$ and outer radius $b$, we can write the magnetic potential $A$ as

$$
\begin{array}{ll}
A=\frac{1}{2} B_{0} r+\frac{q n V_{\theta}}{c} \frac{\ln (b / a)}{b-a} r & \text { for } r \leq a \\
A=\frac{1}{2} B_{0} r+\frac{q n V_{\theta}}{c}\left[r \frac{\ln (b / r)}{b-a}+\frac{1}{2(b-a)} \frac{r^{2}-a^{2}}{r}\right] & \text { for } a \leq r \leq b \\
A=\frac{1}{2} B_{0} r+\frac{q n V_{\theta}}{c} \frac{b+a}{2 r} & \text { for } r>b
\end{array}
$$

when $P_{r}=0, a=b$ and the potential $\psi$ has a minimum at $R$ given by Eq. (2). Thus we expand $\psi$ about $R$, and remembering that $\left.\frac{\partial \psi}{\partial r}\right|_{R}=0$, we have

$$
H=H_{0}+H_{1}=\psi(R)+\frac{1}{2} \frac{\partial^{2} \psi}{\partial r^{2}} x^{2}+\frac{P_{r}^{2}}{2 r m}
$$

or

$$
H_{1}=\frac{P_{r}^{2}}{2 r m}+\frac{1}{2} \frac{\partial^{2} \psi}{\partial r^{2}} x^{2}
$$


where $x$ is the displacement for the equilibrium radius $R$. Taking derivatives of $\psi$ we have

$$
\begin{aligned}
& \frac{\partial \psi}{\partial r}=\frac{1}{r m c^{2}}\left(\frac{P_{\theta}}{r}-\frac{q}{c} A\right)\left(\frac{-P_{\theta}}{r^{2}}-\frac{q}{c} \frac{\partial A}{\partial r}\right) \\
& \frac{\partial^{2} \psi}{\partial r^{2}}=\frac{1}{r m c^{2}}\left[\left(\frac{-P_{\theta}}{r^{2}}-\frac{q}{c} \frac{\partial A}{\partial r}\right)^{2}+\left(\frac{P_{\theta}}{r}-\frac{q}{c} A\right)\left(\frac{2 P_{\theta}}{r^{3}}-\frac{q}{c} \frac{\partial^{2} A}{\partial r}\right)\right]
\end{aligned}
$$

Now since $\left.\frac{\partial \psi}{\partial r}\right|_{R}=0$ we have

$$
\left(\frac{P_{\theta}}{R^{2}}+\frac{Y}{C} \cdot \partial A\right)_{R}=0
$$

Thus

$$
\left.\frac{\partial^{2} \psi}{\partial r^{2}}\right|_{R}=\frac{I}{r m c^{2}}\left(\frac{P_{\theta}}{r}-\frac{q}{c} A\right)_{R}\left(\frac{2 P_{\theta}}{r^{3}}-\frac{q}{c} \frac{\partial^{2} A}{\partial r}\right)_{R}
$$

where

$$
\begin{aligned}
& \left.\frac{\partial A}{\partial r}\right|_{R}=\frac{B_{0}}{2}+\frac{q n V_{\theta}}{c}\left(\frac{1}{b-a}\right)\left(\ln (b / R)-\frac{1}{2}+\frac{a^{2}}{2 R^{2}}\right) \\
& \left.\frac{\partial^{2} A}{\partial r^{2}}\right|_{R}=\frac{q n V_{\theta}}{c(b-a)}\left(-\frac{1}{R}-\frac{a^{2}}{R^{3}}\right)
\end{aligned}
$$

in the 1 imit $b \rightarrow a ; R \rightarrow \frac{b+a}{2}$. Substituting Eq. (2), (A3b) in the limit $b \rightarrow a$, and $(A 10)$ into $(A B)$ we get

$$
\begin{aligned}
\left.\frac{\partial^{2} \psi}{\partial r^{2}}\right|_{R} & =\frac{1}{\gamma m}\left[\frac{(\gamma+v)^{2} m^{2} c^{2}}{R^{2}}-\frac{v^{2} m^{2} c^{2}}{R d}-\frac{(\gamma+v) v m^{2} c^{2}}{R}\left(\frac{1}{R}-\frac{1}{d}\right)\right] \\
& =\gamma m\left[(c / R)^{2}+\left(\frac{v}{\gamma}\right)\left(\frac{c}{R}\right)\left(\frac{c}{R}+\frac{c}{d}\right)\right]
\end{aligned}
$$

where $2 \mathrm{~d}$ is the thickness of the e-layer. Thus the Hamiltonian for the radial motion becomes

$$
H_{1}=\frac{P_{r}^{2}}{2 \gamma m}+\frac{\gamma m}{2} \omega^{2} x^{2}
$$


where

$$
\omega^{2}=(c / R)^{2}\left[1+\frac{v}{\gamma}\left(1+\frac{R}{d}\right)\right]
$$

The condition that $P_{r}^{2} c^{2} \ll r^{2} m^{2} c^{2}$ becomes

$$
\omega^{2} d^{2} \ll c^{2}
$$

or

$$
(d / R)^{2}\left[i+\frac{v}{\gamma}(1+R / D)\right] \Longleftrightarrow 1
$$

when $\omega^{2} d^{2} \sim c^{2}$ then the energy in the radial motion is comparable to that in the azimuthal direction and we will no longer have a well defined e-layer.

The same procedure can be followed to obtain the energy associated with the axial oscillations for an e-layer of finite length. Thus starting with

$$
H_{1}=\frac{P^{2}}{\partial \gamma m}+1 / 2 \frac{\partial^{2} \psi}{\partial z^{2}} \delta^{2}
$$

where

$$
\frac{\partial^{2} \psi}{\partial z^{2}}=-q \frac{\partial^{2} A_{\phi}}{\partial z^{2}}
$$

and $\delta$ is the distance from the center of the layer, with $2 \mathrm{~L}$ being the length of the layer. $A_{\phi}$ differs from the $A$ given previously in that $A_{\phi}$ includes the effects of finite length. Substituting in (A16) we have

$$
H_{1}=\frac{p^{2}}{2 \gamma m}-\frac{q}{2} \frac{\partial^{2} A_{\phi}}{\partial z^{2}} \delta^{2}
$$

The above expression is valid only for $\delta$ small. What is really involved in (A18) is not the second derivative of the potential but the potential difference $A_{\phi}(\delta)-A_{\phi}(0)$. Thus the energy associated with axial oscillations 
is given by

$$
\frac{p^{2}}{2 \gamma m}=-q\left[A_{\phi}(L)-A_{\phi}(0)\right]
$$

If we consider an infinitely thin e-layer the potential can be expressed as follows.

$$
A_{\phi}(z)=\frac{I}{2 L} \frac{R}{c} \int_{-L}^{L} d z^{\prime} \int_{0}^{2 \pi} \frac{d \phi \cos \phi}{\left[2 R^{2}(1-\cos \phi)+\left(z^{\prime}-z\right)^{2}\right]^{1 / 2}}
$$

Where $I$ is the total current, integrating over $z$ ' we have

$$
\begin{aligned}
A_{\phi}(z) & =\frac{I}{2 L C} \int_{0}^{2 \pi} d \phi \cos \phi \\
& \log \left[\frac{L-z+\sqrt{(L-z)+2 R^{2}(1-\cos )}}{L+z+\sqrt{(L+z)^{2}+2 R^{2}(1-\cos \phi)}}\right]
\end{aligned}
$$

Evaluating (A21) at $z=0$, and $L$ we have

$$
\begin{aligned}
& A_{\phi}(0) \equiv 0 \\
& A_{\phi}(L)=\frac{I R}{2 L C} \int_{0}^{2 \pi} d \phi \cos \phi \\
& \quad \log \left[\frac{\sqrt{2 R^{2}(1-\cos \phi)}}{2 L+\sqrt{4 L^{2}+2 R^{2}(1-\cos \phi)}}\right]
\end{aligned}
$$

For $L>R$ we have ${ }^{5}$

$$
\begin{aligned}
& A_{\phi}(L)=\frac{I R}{4 L c} \int_{0}^{2 \pi} d \phi \cos \phi \log (1-\cos \phi) \\
& A_{\phi}(L)=\frac{I R \pi}{2 L c}=-\frac{u m c^{2}}{4 q}
\end{aligned}
$$

Thus we have for $P_{Z}$

$$
\frac{P^{2}}{2 \gamma m}=\frac{u m c^{2}}{4}<\frac{(\gamma m c)^{2}}{2 \gamma m}=\frac{\gamma m c^{2}}{2}
$$

Thus the restriction of $v$ in order that the kinetic energy associated with axial oscillations be less than $\mathrm{rmc}^{2}$ is

$$
\nu<2 \gamma
$$




\section{APPENDIX B}

Here we consider the power spectrum of the synchrotron radiation emitted from a single elctron in a non-current-neutralized e-layer. If we assume a linear change in the B-field across the e-layer we can express the magnitude of the B-field as a function of its position in the layer as

$$
B(x)=B_{0}\left(\frac{\gamma-v}{\gamma+v}+\frac{2 v x}{\gamma+v}\right)
$$

the power radiated, $P$, is proportional to $B^{2}$ thus we have

$$
P(x)=P_{0}\left(\frac{\gamma-v+2 v x}{\gamma+v}\right)^{2}
$$

and the average power is given by

$$
\begin{aligned}
\langle P\rangle & =P_{0} \int_{0}^{1}\left(\frac{\gamma-v+2 v x}{\gamma+v}\right)^{2} d x \\
& =p_{0} \frac{\gamma^{2}+v^{3} / 3}{(\gamma+v)^{2}} .
\end{aligned}
$$

where the subscript 0 refers to the quantity subscripted in the currentneutralized system of the same e-layer energy and with magnetic field $B_{0}$.

To calculate the spectrum it is necessary to average the spectrum for an electron in a given magnetic field over the range of the magnetic field in the e-layer. This is done as follows, define

$$
\left.I_{0}(\omega)=\int_{0}^{1}: x d x I(\omega, \omega)(x)\right)
$$

where $I\left(11, v_{0}(1) c(x)\right)$ is given by 3,5

$$
I\left(\omega, \omega_{c}(x)\right)=(2 \sqrt{3})\left(\omega / \omega_{c}\right)\left(e^{2} \gamma / c\right) \int_{2\left(\omega / \omega_{c}\right)}^{\infty} K_{5 / 3}(y) d y
$$


and

$$
\omega_{c}(x)=\frac{3 e B_{0} \gamma^{3}}{m c \sqrt{\gamma^{2}-1}}\left[\frac{\gamma-\nu}{\gamma+\nu}+\frac{2 v x}{\gamma+v}\right]
$$

$I\left(\omega, \omega_{c}\right)$ is the energy radiated per unit frequency interval per revolution, as a function of the local $\omega_{0}$ and $\omega_{c}$. We can compare the effects of current neutralization on the radiation spectrum by fixing $\gamma$ and $R$, and allowing $v$ to vary in Eq. (B6). Defining ${ }^{B}{ }_{C N}$ as the magnetic field in the current neutralized system, $B_{0}$ is given by

$$
{ }^{B}={ }^{B}{ }_{C N}\left(\frac{y+v}{\gamma}\right)
$$

and $\omega_{c}(x)$ in terms of the current neutralized critical frequency ${ }^{\omega} \mathrm{CN}$ is given by

$$
\omega_{C}(x)=\omega_{C N}[1+v / \gamma(2 x-1)]
$$

The average power $\langle\mathrm{P}\rangle$ in terms of $\mathrm{P}_{\mathrm{CN}}$ is given by

$$
\begin{aligned}
& \langle P\rangle=P_{C N} \int_{0}^{1}[1+v / \gamma(2 x-1)]^{2} d x \\
& \cdot\langle P\rangle=P_{C N} \frac{\gamma^{2}+v^{2} / 3}{\gamma^{2}}
\end{aligned}
$$

Equation (B4) was integrated numerically to yield the radiation spectrum in Fig. 1.6 


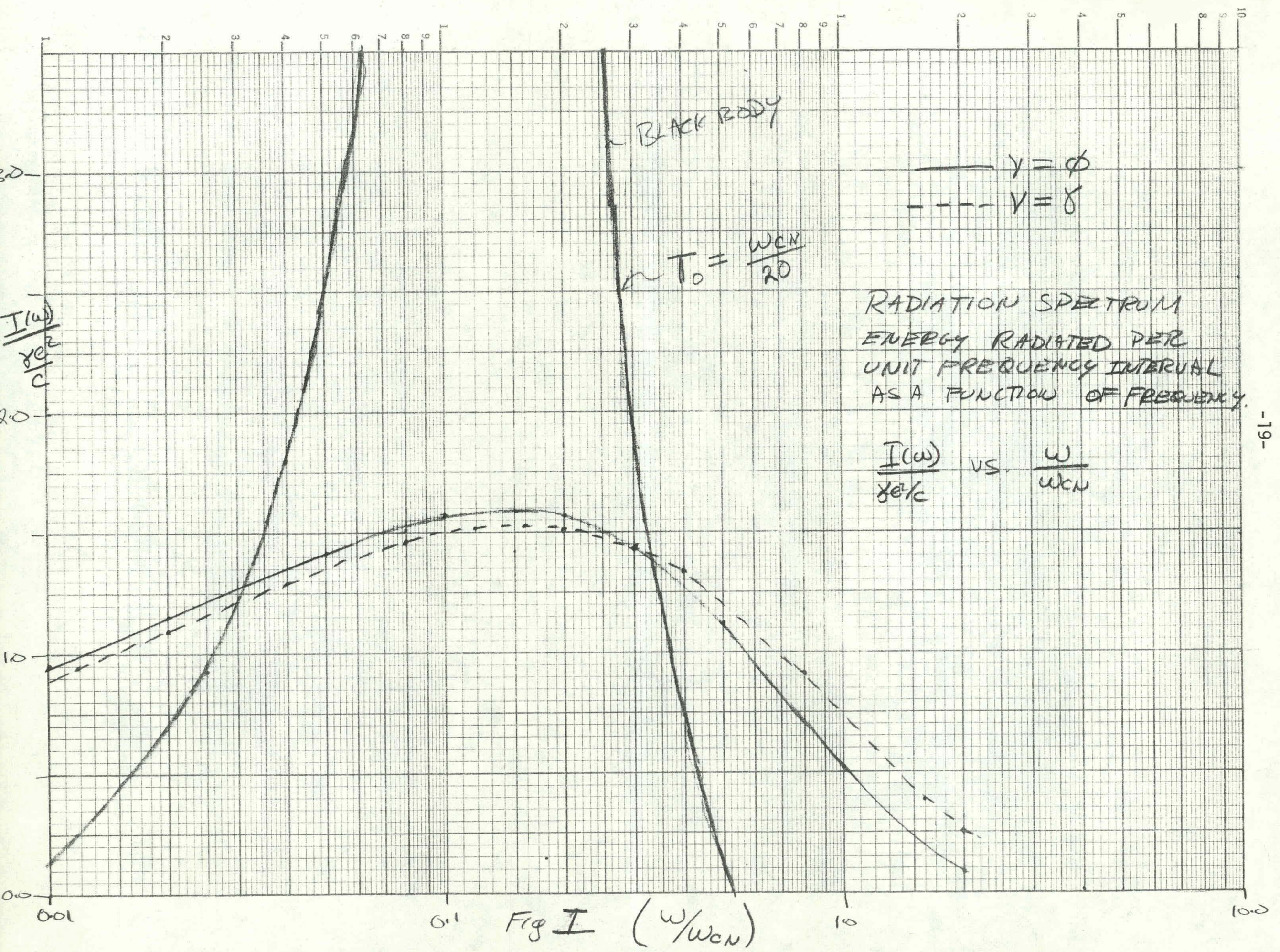




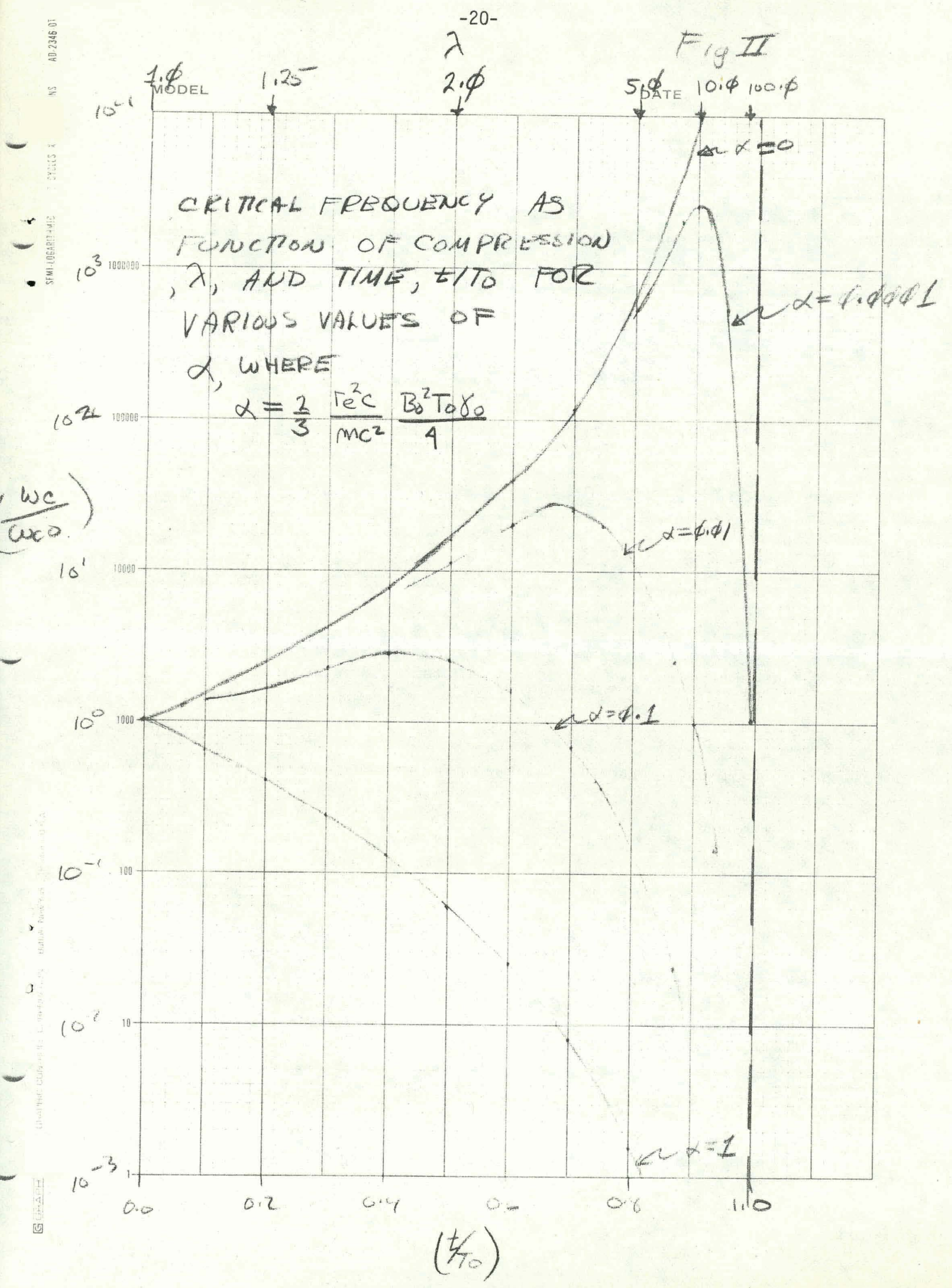




\section{REFERENCES}

1. Nowak, D. and Shearer, J, COPD 74-59.

2. Schmidt, G., The Phys. of Fluids, Vol. 15, No. 8, 1972.

3. See for example Jackson, J. D., Classical Electrodynamics.

4. See for example Alfven, H., Phys. Rev, Vol. 55, No. 5, 1939.

5. Garelis, E., private comunication.

6. Garelis, E., private communication. 
DISTRIBUTION

\section{External}

John Farber

Peter Haas

David Oakley

Gordon Soper

Defense Nuclear Agency

Washington, D.C, 20305

Dr. Jack Rosengren

Dr. B. Hartenbaum

Dr. C. A. McDonald

$R$ \& D Associates

Santa Monica, California 90403

Dr. L. Levine

Dr. A. E. Robson

Dr. P. Turchi

Naval Research Laboratory

Washington, D. C. 20375

\section{Internal}

W. C. Condit $(A / L-33)$

E. K. Freytag (EE/L-153)

E. Garel is $(A / L-33)$

B. C. Groseclose (Director's office/L-2)

W. A. Lokke $(A / L-33)$

D. A. Nowak $(A / L-33)$

J. W. Shearer $(M / L-387)$

P. C. Wheeler (Nuclear Explosives/L-21)

TID Files (L-9) (5)

A-Division Files (L-33) (15)

"This report was prepared as an account of work sponsored by the United States Government. Neither the United States nor the United States Atomic Energy Commission, nor any of their employees, nor any of their contractors, subcontractors, or their employees, makes any warranty, express or implied, or assumes any legal liability or responsibility for the accuracy, completeness or usetulness of any intormation, apparatus, product or process disclosed, or represents that its use would not infringe privately. owned rights." 